\title{
Individual and sex differences in reminiscence
}

\author{
KUO LONG HUANG and R. B. PAYNE \\ University of Georgia, Athens, Georgia 30602
}

\begin{abstract}
Inverted alphabet printing, rotary pursuit, and mirror tracking tasks were administered to 84 subjects in order to ascertain (a) reproducibility of reminiscence scores within and between tasks and (b) sex differences in reminiscence. With prerest performance levels held constant by second-order partial correlation procedures, reproducibility of individual reminiscence differences within tasks was significant but quite low, while predictability of reminiscence from one task to another was negligible. The sexes reminisced essentially alike on inverted alphabet printing, but females reminisced more than males on the other tasks, presumably because they were relatively more depressed by massed practice on these tasks. Thus, individual and sex differences were essentially task specific. Implications of results for reminiscence theories and for the credibility of alleged relationships between reminiscence and other organismic variables were discussed.
\end{abstract}

Reminiscence is one of the most visible phenomena in the whole field of perceptual motor skills. Although reminiscence scores are typically quite variable, the average effect appears with monotonous consistency and clarity whenever the appropriate experimental operations are conducted. As an average effect, it bears an orderly and reproducible relationship to various antecedent conditions such as the length of the rest period (Kimble \& Horenstein, 1948) and the amount of prerest practice (Ammons, 1947; Irion, 1949). Its empirical status is well established and seemingly unassailable.

Reminiscence has also played an important, but more controversial, role in various theoretical developments. For example, Hull (1943) viewed reminiscence as an index of the hypothetical factor called reactive inhibition $\left(I_{R}\right)$; the subsidence of which was said to account for the well-known effects of practice distribution. Within the personality domain, Eysenck (1956) used reminiscence measures for the purpose of testing propositions about the differential susceptibility of extroverts and introverts to $I_{R}$.

Both Hull and Eysenck, like nearly everyone else, assumed that reminiscence tendencies were stable, reproducible characteristics of organisms. Indeed, both positions required such an assumption. By implication, at least, both also assumed corresponding differences in the "hypothetical factors responsible for reminiscence. Hull (1945) was particularly clear on this point when he asserted that the forms of equations representing behavioral laws were the same from one individual to another even though differences might appear in the empirical constants. Later Hull (1951) seemed less certain about the stability of such differences from one occasion to another, but he continued to regard them as

This paper is based on a thesis submitted by the senior author in partial fulfillment of requirements for the MS degree at the University of Georgia. Reprints may be obtained from $R$. $B$. Payne, Department of Psychology, University of Georgia, Athens, Georgia 30602. something apart from the purely random events which comprised behavioral oscillation $\left({ }_{S} \mathrm{O}_{R}\right)$.

Despite the importance of assumptions about the consistency of reminiscence scores, there have been surprisingly few attempts to measure it within any given task; and the authors found no attempts at all to discover the predictability of reminiscence on one task from a similar measure on another. Eysenck (1956), for example, found a correlation of .44 between two sets of crude gain scores on rotary pursuit. Finding this value somewhat disappointing for his own purposes, he ascribed it to the unreliability of brief postrest samples, the second of which, he alleged, was particularly contaminated with error variance arising from the extinction of conditional inhibition. Although Eysenck (1965) eventually abandoned inhibition in favor of consolidation as the primary explanation of reminiscence, his requirement for reproducible individual differences in reminiscence remained. The likelihood of finding such differences was seriously disparaged by Peters $(1972,1973)$, who found no true individual differences at all in reminiscence as conventionally measured.

The purpose of the present study was to ascertain the reproducibility of reminiscence scores within and between three commonly used testing situations. An ancillary purpose was to explore sex differences in reminiscence, the empirical status of which also appears uncertain (Ammons, Alprin, \& Ammons, 1955; Archer, 1958). The factual status of these and kindred matters is obviously germane to reminiscence theories and to the credibility of alleged relationships between reminiscence and other organismic variables.

\section{METHOD}

\section{Subjects}

The sample consisted of 84 subjects ( 42 males, 42 females) enrolled in introductory psychology courses. All were Caucasian and right-handed, and their modal age was 18 years. All received course credit points for participation. 
Table 1

Correlations* Among Reminiscence Scores Expressed as Crude Gains

\begin{tabular}{lrrr}
\hline & RP & MT & AP \\
\hline Zero Order & & & \\
$\quad$ Rotary Pursuit $\dagger$ & .12 & -.06 & .01 \\
Mirror Tracking & & .03 & -.08 \\
$\quad$ Alphabet Printing & & & .17 \\
Second-Order Partial & & & \\
$\quad$ Rotary Pursuit & .40 & .11 & .08 \\
Mirror Tracking & & .32 & .01 \\
$\quad$ Alphabet Printing & & & .43 \\
\hline
\end{tabular}

$* A$ correlation of .24 is required for significance at $p=.05$. tThirty-second scores.

\section{Tasks}

Inverted alphabet printing $(A)$, rotary pursuit $(R)$, and mirror tracking (M) comprised the performance battery. The alphabet task, similar to that used by Kimble (1949), required the subject to print the capital letters upside down so that they could be read normally when the paper was turned $180 \mathrm{deg}$. Score was the number correct/minute. The rotary pursuit task was provided by the standard USAF Rotary Pursuit Test, Model CM803B2, as described by Melton (1947). Time on target was recorded in units of $.01 \mathrm{sec}$ for each 30 -sec period of practice. The mirror tracking task (Payne \& Artley, 1972) required subjects to use mirror vision in order to keep a stylus on a small silver target as it moved clockwise at $1 \mathrm{rpm}$ through a narrow star-shaped pathway. Time on target was recorded for each minute of practice.

\section{Procedure}

The 84 subjects were assigned in equal sex numbers to three orders of the foregoing tasks (MAR, ARM, RMA). Each subject executed a sequence consisting of 3-min massed practice, 3-min rest, 3-min massed practice, 3-min rest, and 3-min massed practice, with $3-\mathrm{min}$ transit time between tasks.

\section{RESULTS}

Reminiscence scores were computed in two ways. The first method yielded simple differences between terminal prerest and initial postrest scores. These are called crude gain scores. The second method took some account of the practice effect of the last prerest trial by extrapolating the linear component of the prerest trend for each subject and using the extrapolated score as the base for computing reminiscence. These values are called estimated gain scores.

\section{Consistency of Reminiscence Scores}

Crude gain scores. Correlations among reminiscence scores within and between tasks were derived from the error terms of variance-covariance analyses in which orders and sexes comprised the main effects. With prerest performance levels uncontrolled, as in Eysenck (1956), the zero-order correlations within tasks ranged from .03 to .17 , as shown in Table 1. One can see also that the correlations between first-period scores across tasks were negligible. Since reminiscence scores were correlated negatively with prerest performance levels,
Table 2

Correlations* Among Reminiscence Scores Expressed as Estimated Gains

\begin{tabular}{lllr}
\hline & RP & MT & AP \\
\hline Zero Order & & & \\
$\quad$ Rotary Pursuit $\dagger$ & .12 & .01 & .07 \\
Mirror Tracking & & .25 & -.17 \\
$\quad$ Alphabet Printing & & & .14 \\
Second-Order Partial & & & \\
$\quad$ Rotary Pursuit $\dagger$ & .52 & .28 & .22 \\
$\quad$ Mirror Tracking & & .66 & .03 \\
Alphabet Printing & & & .66 \\
\hline
\end{tabular}

${ }^{*} A$ correlation of .24 is required for significance at $p=.05$. tThirty-second scores.

the contamination of reminiscence variance by performance variance was minimized by the computation of second-order partial correlations between reminiscence scores with prerest levels held constant. As seen in the lower half of Table 1, the correlations were somewhat improved by this procedure, but they remained weak. At best, the common variance of two sets of scores from the same task was about $18.5 \%$, and the common variance of two sets of scores, each from a different task, was about $1 \%$. Prudence compels the conclusion that these different sets of scores were largely measures of different things.

Estimated gain scores. With prerest performance levels uncontrolled, the zero-order correlations between reminiscence scores within tasks ranged from .12 to .25 , while those between first reminiscence scores across tasks ranged from -.17 to .07 , as shown in the top half of Table 2. When estimated prerest levels were controlled by second-order partial correlation procedures, within-test correlations ranged from .52 to .66 , while between-test correlations ranged from .03 to .28 , as seen in the bottom half of Table 2 . Although some improvement in the apparent consistency of the scores can be noted, particularly within tests, the picture remained far from satisfactory by any reasonable psychometric standard. Much of this improvement over the partial correlations among crude gain scores probably resulted from large variance increases attributable to the computational method. Thus, the estimated gain scores, like the crude gain scores, were simply not very reproducible even within the same task.

\section{Sex Differences in Reminiscence Scores}

Within each task, the 168 crude gain scores were subjected to a mixed-model variance analysis in which orders and sexes comprised the main between-subjects effects and reminiscence periods the main within-subjects effect. Also, in order to complement the reminiscence findings, the 7561 -min performance scores on each test were subjected to a mixed-model variance analysis in which orders and sexes comprised the main between-subjects effects and trial blocks and trials 


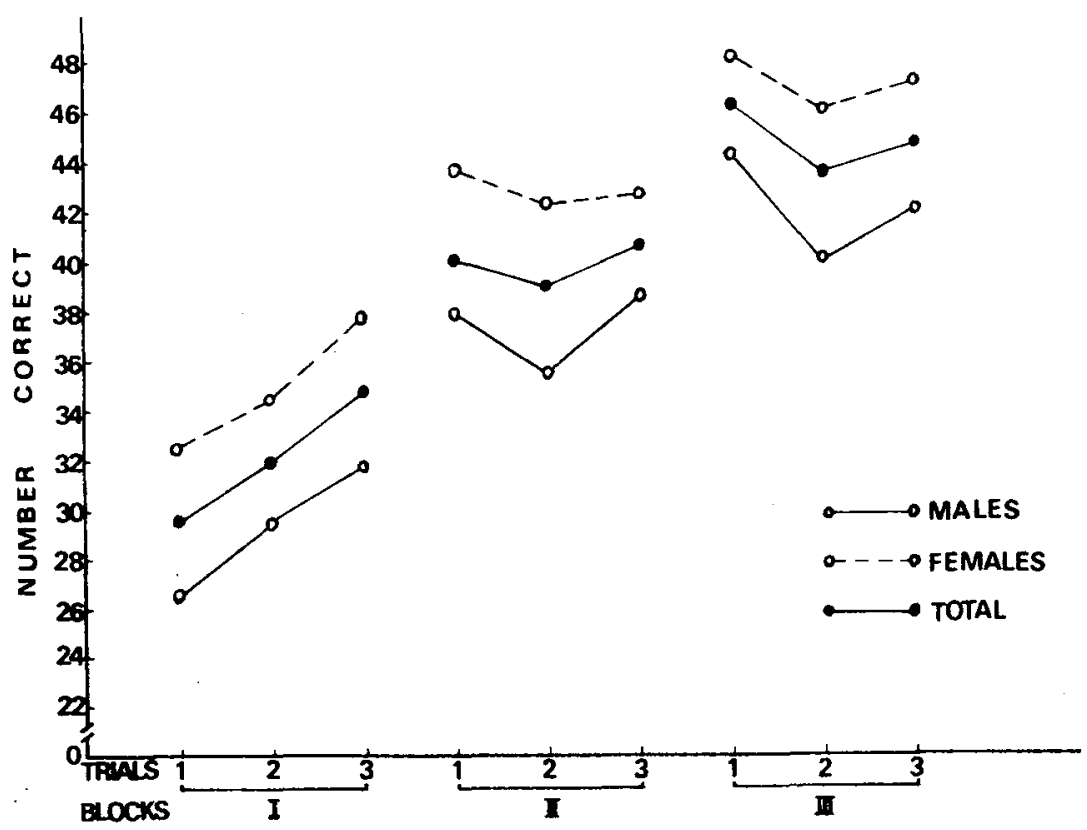

Figure 1. Inverted alphabet printing performance trends.

within blocks the main within-subjects effects.

Inverted alphabet printing. Although reminiscence clearly occurred during both rest periods in this task, as shown in Figure 1, there was no significant variation attributable to orders, sexes, periods, or their interactions $(F<1)$. There were, however, several significant sources of variation in performance. Among these, block, trial, and BT effects, although significant $(p<.001)$, were of limited interest. The more relevant effects were associated with orders $[F(2,78)=3.74$, $\mathrm{p}<.05]$, sexes $[\mathrm{F}(1,78)=6.97, \mathrm{p}<.01]$, and the SBT interaction $[F(4,312)=3.18, p<.025]$. Despite the significance of the triple interaction, females surpassed males on every combination of trial and block, as shown in Figure 1. An analysis of the order effect showed that poorest performance occurred when the task was in last position, but this effect was orthogonal to all others. Thus, although females performed consistently better than males, the two sexes reminisced essentially alike.

Rotary pursuit. Analysis of reminiscence scores on rotary pursuit revealed a significant sex effect $[F(1,78)=$ $6.63, p<.025]$ and a significant Sexes by Periods interaction $[F(1,78)=5.90, p<.025]$. An analysis of this interaction showed that females reminisced significantly more than males only during the first rest period $(p<.025)$. Means: $\mathrm{MP}_{1}=10.9, \mathrm{MP}_{2}=8.12$, $\mathrm{FP}_{1}=15.3, \mathrm{FP}_{2}=8.44$. There was also a significant period effect $[F(1,78)=32.95, p<.001]$, but this was more prominent for females than for males. Analysis of performance levels, shown in Figure 2, revealed several
Figure 2. Rotary pursuit performance trends.

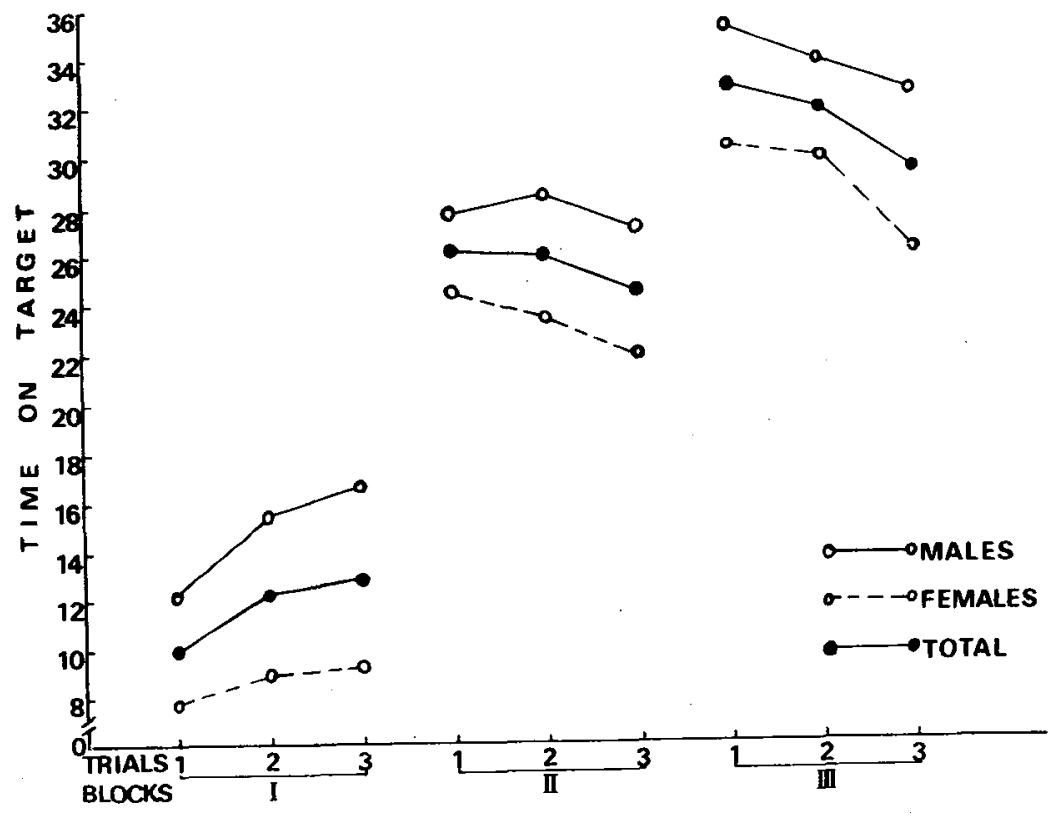




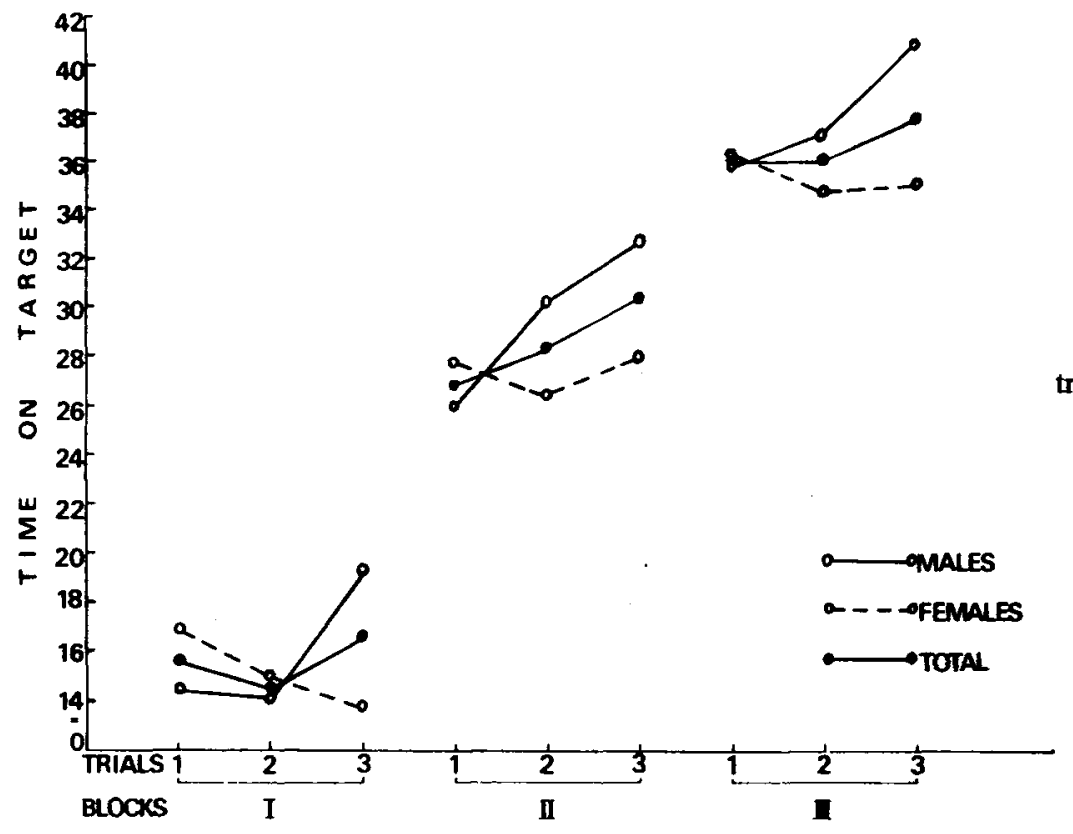

significant sources of variation. Among these, block, trial, and BT effects, although significant $(p<.005)$, were of marginal interest. The more relevant effects were associated with sexes $[F(1,78)=9.21, p<.005]$ and the Sexes by Trials interaction $[F(2,156)=6.68, p<.005]$. Although males were consistently superior to females across trials, as usually found (Noble, 1970), the sex differences increased as trials progressed, i.e., males gained proficiency slightly across trials, while females lost proficiency. Means: $\mathrm{MT}_{1}=25.11, \mathrm{FT}_{1}=20.92$, $\mathrm{MT}_{2}=25.95, \mathrm{FT}_{2}=20.83, \mathrm{MT}_{3}=25.51, \mathrm{FT}_{3}=19.12$. Thus, females were relatively more depressed by practice massing, as one might have suspected from the analysis of reminiscence differences. Since Noble (1970) also found a significant ST interaction in rotary pursuit, the question arises whether sex differences in performance on this task, and perhaps others, depend upon procedure as well as sex-linked differences in basic aptitude.

Mirror tracking. Analysis of reminiscence scores on mirror tracking revealed that females reminisced more than males regardless of period $[F(1,78)=18.60$, $\mathrm{p}<.001]$. Means: $\mathrm{MP}_{1}=6.54, \quad \mathrm{FP}_{1}=13.91$, $\mathrm{MP}_{2}=3.12, \mathrm{FP}_{2}=8.18$. There was also a significant period effect $[F(1,78)=10.54, p<.005]$, but this was orthogonal to the sex effect. The sex difference is especially well clarified by the performance trends shown in Figure 3. Here one can perceive a remarkable Sex by Trial interaction $[F(2,156)=16.66, p<.001]$. In fact, given the obvious similarity of sex means at the start of each practice block ( $p>.05$ ), one might almost say that the sex difference was produced by practice massing. At any rate, it is quite clear that females were relatively more depressed by massed practice than males, as in rotary pursuit.
Figure 3. Mirror tracking performance trends.
The foregoing results obviously comprise a mixed bag when viewed in terms of traditional reminiscence theory. The average reminiscence effect appeared with faithful regularity, as it nearly always does. However, the alignments of individual differences across repeated measures were very poor even within tasks, and they were essentially random when viewed across tasks. The arrays of differences appeared to have been determined largely by a fortuitous interplay of subject factors and factors that were peculiar not only to the tasks in which the differences were generated but also to the separate measurement occasions themselves. Sex group differences were slightly more consistent, but they were also largely task bound. These are essentially the same conclusions reached by Roff and Payne (1956) in their search for rational bases from which to project intercorrelations among crude gain scores as indicants of learning ability.

As far as inhibition theory is concerned, reminiscence behaves more like an index of Hull's behavioral oscillation than an index of reactive inhibition. Hull $(1943,1945)$, of course, regarded the accumulation and dissipation of $I_{R}$ as orderly, predictable functions of specified antecedent conditions in the individual case as well as the group case. However, the correlations reported above say that this cannot be assumed for the individual case. If the accumulation function is lawful for individual cases, then the dissipation function cannot be lawful. If the dissipation function is lawful for individual cases, then the accumulation function cannot be lawful. Perhaps neither is lawful for individual cases. If that is so, how can the average phenomenon be as lawful and predictable as it is? The authors see no 
fundamental incompatibility between group and individual cases, even if reminiscence, like oscillation, is a random variable. Consider Hull's (1943) expression of effective reaction potential $\left(s \bar{E}_{R}\right)$. Other factors being equal, response level is some function of ${ }_{S} \bar{E}_{R}$ which, in turn, is the algebraic sum of a positive-factor reaction potential $\left(\mathrm{s}_{\mathrm{R}}\right)$ and a negative-factor reactive inhibition $\left(I_{R}\right)$. In other words, $s \bar{E}_{R}={ }_{S} E_{R}+\left(-I_{R}\right)$. During rest $I_{R}$ subsides and performance rises. In other words, ${ }_{s} E_{R}={ }_{s} \bar{E}_{R}-\left(-I_{R}\right)$. Now, if one assumes that (a) the amount of $I_{R}$ generated in a given trial is determined by a fortuitous interplay of subject factors and task factors (e.g., duration and effortfulness of practice), (b) these amounts accumulate across massed trials, and (c) the amount dissipated during rest is limited only by the amount accumulated and the time available, one can reproduce the typical reminiscence effect by using a table of random numbers to assign increments and decrements of $I_{R}$ to individual subjects. Thus, the average effect can be achieved despite the complete lack of reproducibility of individual reminiscence values. The typical effect of practice distribution can also be reproduced in this way.

Finally, the marginal reproducibility of individual differences in reminiscence within tasks casts further doubt upon the viability of Eysenck's notions about reminiscence and personality. Moreover, this doubt is potentiated by negligible correlations among reminiscence scores across tasks. Given the prospect of $\mathrm{rem}_{\mathrm{a}}, \mathrm{rem}_{\mathrm{b}}, \mathrm{rem}_{\mathrm{c}}$, and perhaps $\ldots \mathrm{rem}_{\mathrm{n}}$, where the subscripts refer to tasks, the theory must somehow rationalize the choice of tasks before it can be taken seriously.

\section{REFERENCES}

Ammons, R. B. Acquisition of motor skill: II. Rotary pursuit performance with continuous practice before and after a single rest. Journal of Experimental Psychology, 1947, 37, 393-411.

Ammons, R. B., Alprin, S. I., \& Ammons, C. H. Rotary pursuit performance as related to sex and age of pre-adult subjects. Journal of Experimental Psychology, 1955, 49, 127-133.

Archer, E. J. Effect of distribution of practice on a component skill of rotary pursuit tracking. Journal of Experimental Psychology, 1958, 56, 427-436.

Eysenck, H. J. Reminiscence, drive, and personality theory. Journal of Abnormal and Social Psychology, 1956, 53, 328-333.

Eysenck, H. J. A three-factor theory of reminiscence. British Journal of Psychology, 1965, 56, 163-181.

Hull, C. L. Principles of behavior. New York: Appleton-Century-Crofts, 1943

Hull, C. L. The place of innate individual and species differences in a natural-science theory of behavior. Psychological Review, $1945,52,55-60$.

Hull, C. L. Essentials of behavior. New Haven: Yale University Press, 1951.

Irion, A. L. Reminiscence in pursuit-rotor learning as a function of rest and amount of pre-rest practice. Journal of Experimental Psychology, 1949, 39, 492-499.

Irion, A. L. Historical introduction. In E. A. Bilodeau (Ed.), Principles of skill acquisition. New York: Academic Press, 1969.

Kimble, G. A. An experimental test of a two-factor theory of inhibition. Journal of Experimental Psychology, 1949, 39, 15-23.

Kimble, G. A., \& Horenstein, B. Reminiscence as a function of the amount of interpolated rest. Journal of Experimental Psychology, 1948, 38, 239-244.

Melton, A. W. (Ed.) A pparatus tests. Washington, D.C: U.S. Government Printing Office, 1947.

Noble, C. E. Acquisition of pursuit tracking skill under extended training as a joint function of se $x$ and initial ability. Journal of Experimental Psychology, 1970, 86, 360-373.

Payne, R. B., \& Artley, C. W. Facilitation of psychomotor learning by classically differentiated supplementary feedback cues. Journal of Motor Behavior, 1972, 4, 47-55.

Peters, E. N. Nonexistent individual differences in reminiscence. Psychological Bulletin, 1972, 78, 375-378.

Peters, E. N. A note on alleged individual differences in reminiscence and extraversion-introversion. Psychological Bulletin, 1973, 80, 245-246.

Roff, M. \& Payne, R. B. The relation between learning ability scores obtained in different situations. Journal of Psychology, $1956,41,47-54$. 